\title{
EXIT-constrained BICM-ID design using extended mapping
}

Kisho Fukawa ${ }^{*}$, Soulisak Ormsub ${ }^{1}$, Antti Tölli ${ }^{2}$, Khoirul Anwar ${ }^{1}$ and Tad Matsumoto ${ }^{1,2}$

\begin{abstract}
This article proposes a novel design framework, EXIT-constrained binary switching algorithm (EBSA), for achieving near Shannon limit performance with single parity check and irregular repetition coded bit interleaved coded modulation and iterative detection with extended mapping (SI-BICM-ID-EM). EBSA is composed of node degree allocation optimization using linear programming (LP) and labeling optimization based on adaptive binary switching algorithm jointly. This technique achieves exact matching between the Demapper (Dem) and decoder's extrinsic information transfer (EXIT) curves while the convergence tunnel opens until the desired mutual information (MI) point. Moreover, this article proposes a combined use of SI-BICM-ID-EM with Doped-ACCumulator (D-ACC) and modulation doping (MD) to further improve the performance. In fact, the use of D-ACC and SI-BICMID (noted as DSI-BICM-ID-EM) enables the right-most point of the EXIT curve of the combined demapper and D-ACC decoder $\left(D_{\text {dacc }}\right)$, denoted as DemD dacc, to reach a point very close to the $(1.0,1.0)$ MI point. Furthermore, MD provides us with additional degree-of-freedom in "bending" the shape of the demapper EXIT curve by choosing the mixing ratio of modulation formats, and hence the left most point of the demapper EXIT curve can flexibly be lifted up/pushed down with MD aided DSI-BICM-ID-EM (referred to as MDSI-BICM-ID-EM). Results of the simulations show that near-Shannon limit performance can be achieved with the proposed technique; with a parameter set obtained by EBSA for MDSI-BICM-ID-EM, the threshold signal-to-noise power ratio (SNR) is only roughly $0.5 \mathrm{~dB}$ away from the Shannon limit, for which the required computational complexity per iteration is at the same order as a Turbo code with only memory-2 convolutional constituent codes.
\end{abstract}

\section{Introduction}

The discovery of Turbo code [1] in 1993 is a landmark event in the history of coding theory, since the code can achieve near-Shannon limit performance. It is shown in [1] that the Turbo code composed of memory-4 constituent convo-lutional codes can achieve $0.7 \mathrm{~dB}$, in Signal-tonoise power ratio (SNR), away from the Shannon limit. Various efforts have been made since then to achieve Turbo code-like performance without requiring heavy computational efforts for decoding.

Bit-interleaved coded modulation and iterative detection/decoding (BICM-ID) [2] has been recognized as a bandwidth efficient coded modulation scheme, of which transmitter is comprised of a concatenation of encoder and bit-to-symbol mapper separated by a bit interleaver. Iterative detection-and-decoding takes place at the

\footnotetext{
* Correspondence: kisho.fukawa@gmail.com

${ }^{1}$ School of Information Science, Japan Advanced Institute of Science and Technology (JAIST), 1-1 Asahidai, Nomi, Ishikawa, 923-1292, Japan Full list of author information is available at the end of the article
}

receiver, where extrinsic log likelihood ratio (LLR), obtained as the result of the maximum a posteriori probability (MAP) algorithm for demapping/decoding, is forwarded to the decoder/demapper via de-interleaver/ interleaver and used as the a priori LLR for decoding/ demapping according to the standard turbo principle.

Performances of BICM-ID have to be evaluated by the convergence and asymptotic properties [3], which are represented by the threshold SNR and bit error rate (BER) floor, respectively. In principle, since BICM-ID is a serially concatenated system, analyzing its performances can rely on the area property [4] of the EXtrinsic Information Transfer (EXIT) chart. Therefore, the transmission link design based on BICM-ID falls into the issue of matching between the demapper and decoder EXIT curves.

Various efforts have been made seeking for better matching between the two curves to minimize the gap, while still keeping the tunnel open, aiming, without requiring heavy detection/decoding complexity, at

\section{实}


achieving lower threshold SNR and BER floor. In [5], ten Brink et al. introduced a technique that makes good matching between the detector and decoder EXIT curves using low density parity check (LDPC) code in multiple input multiple output (MIMO) spatial multiplexing systems.

It has long been believed that for 4-quadrature amplitude modulation (4-QAM), the combination of Gray mapping and Turbo or LDPC codes achieves the optimal performance. However, Schreckenbach et al. [6] propose another approach towards achieving good matching between the two curves by introducing different mapping rules, such as non-Gray mapping, which allows the use of even simpler codes to achieve BER pinch-off (corresponding to the threshold SNR) at an SNR value relatively close to the Shannon limit.

Another technique that can provide us with the design flexibility is extended mapping (EM) presented in $[7,8]$ where with $2^{m}$-QAM, $\ell_{\text {map }}$ bits $\left(\ell_{\text {map }}>m\right)$, are allocated to one signal point in the constellation. With EM, the left-most point of the demapper EXIT function has a lower value than that with the Gray mapping, but the right-most point becomes higher. With this setting, the demapper EXIT function achieves better matching even with weaker codes such as short memory convolution codes as shown in [7]. However, there is a fundamental drawback with the structure shown in [7]; it still suffers from the BER floor simply because the demapper EXIT curve does not reach the top-right $(1.0,1.0)$ MI point.

In [9], Pfletschinger and Sanzi suggest that by using the memory-1 rate-1 recursive systematic convolutional code (RSCC), referred to as D-ACC located immediately after the interleaver, the error floor can be eliminated. Furthermore, it was shown by [10] that by replacing the RSCC-coded bits $b_{u}(P)$ with the accumulated bits $b_{c}(P)$ at every $P$ bit-timings, the technique of which is referred to as inner doping with doping ratio $(1: P)$, the EXIT curve of $D e m D_{\text {dacc }}$ can be flexibly changed.

Several techniques have been proposed to determine optimal labeling pattern for the modulation (bit pattern vector allocated to each constellation point). The ideas of binary switching algorithm (BSA), which aims at labeling costs optimization, are presented in $[6,11]$. However, the BSA based labeling optimization evaluates the labeling cost assuming that full a priori information is available. Hence, this approach only aims at lifting up as much the rightmost point of the demapper EXIT curve as possible. Yang et al. [12] introduce adaptive binary switching algorithm (ABSA) to obtain optimal labeling pattern, where optimality is defined by taking into account the labeling costs at multiple a priori MI points. Hence, ABSA changes the shape of the demapper EXIT curves more flexibly than BSA. However, the optimal labeling obtained in ABSA is on given code- basis since the code parameter optimization is not included in the ABSA iterations.

In our previous publication [13], we introduced a BICM-ID technique that uses even simpler codes, single parity check code (SPC) and irregular repetition code (IRC), combined with EM. For the notation simplicity, we refer our proposed BICM-ID structure in [13] to as SPC-and-IRC aided BICM-ID with EM (SI-BICM-IDEM). We investigated in [14] that linear programming (LP) technique can be applied for SI-BICM-ID-EM to determine the optimal degree allocations for the IRC code with the aim of achieving desired convergence property. Moreover, in [15] we proposed a combined use of modulation doping (MD), originally proposed in $[16,17]$, which mixes the labeling rules for the extended non-Gray mapping and the standard Gray mapping at a certain ratio. The technique proposed in [15] helps the left-most point of the demapper slightly be lifted up to initiate the LLR exchange between the demapper and the decoder. This technique gives the additional degreeof-freedom in "bending" the shape of the demapper EXIT curve by choosing the mixing ratio and hence the left-most point of the demapper EXIT curve can be flexibly lifted up/pushed down. This article proposes a combined use of SI-BICM-ID-EM with D-ACC and MD. The D-ACC aided SI-BICM-ID-EM is referred to as DSI-BICM-ID-EM, and MD aided DSI-BICM-ID-EM is referred to as MDSI-BICM-ID-EM later on.

The primary goal of this article is to create a design framework for the optimization of SI-BICM-ID-EM ${ }^{a}$ by combining those techniques into a unified iterative algorithm. To achieve the goal described above, this article proposes a new labeling pattern optimization technique, EXIT-constrained Binary Switching Algorithm (EBSA). The gap between the two EXIT curves is taken into account in a repeat-until loop that controls the EBSA algorithm. Hence, the process for determining the optimal degree allocation using LP [13,14] is also included in the repeat-until loop in EBSA.

The results of simulations show that near-Shannon limit performance can be achieved with the proposed techniques; BER simulation results show that 4-QAM $E M$ with $\ell_{\text {map }}=5$, the threshold SNR is only roughly $0.5 \mathrm{~dB}$ away from the Shannon limit with MDSI-BICMID-EM, for which required computational complexity for $D e m D_{\text {dacc }}$ is almost the same as a Turbo code with only memory- 2 convolutional constituency codes, per iteration.

This article is organized as follows; our proposed system structure is described in Section 2. Theoretical EXIT functions of the codes used in SI-BICM-ID-EM are presented in Section 3. EBSA is introduced and detailed in Section 4, which is the core part of this contribution. In Section 5 numerical results are provided: in 
Section 5.1, convergence property of the proposed schemes described to confirm the effectiveness of EBSA; in Section 5.2, the results of BER performance evaluations are presented. In Section 6, computational complexity with the proposed technique is assessed briefly. Finally, we conclude this article in Section 7 with some concluding statements.

\section{System model}

\subsection{Transmitter}

Figure 1 describes the system model considered in this article. The SI-BICM-ID-EM technique, which this article is based on, is detailed in [13] including its schematic diagram. Therefore, it is only summarized in this section. The binary bit information sequence $u$ to be transmitted is encoded by, first, a single parity check code where a single parity bit is added to every $d_{c}-1$ information bits, followed by a repetition code. $d_{c}$ is referred to as check node degree.

The repetition times $d_{v}$, referred to as variable node degree, may take different values in a block (transmission frame); if $d_{v}$ takes several different values in a block, such code is referred to as having irregular degree allocations. It is assumed throughout this article that $d_{c}$ takes only one identical value as in [5].

The rate of the code is

$$
R=\frac{\left(d_{c}-1\right)}{d_{c} \sum_{i=1}^{M}\left(a_{i} \cdot d_{v_{i}}\right)}
$$

and the spectrum efficiency is

$$
\begin{aligned}
\eta & =\ell_{\text {map }} \cdot R \\
& =\frac{\ell_{\text {map }} \cdot\left(d_{c}-1\right)}{d_{c} \sum_{i=1}^{M}\left(a_{i} \cdot d_{v_{i}}\right)}
\end{aligned}
$$

bits per symbol, where $a_{i}$ represents the ratio of variable nodes having degree $d_{v_{i}}$ in a block and $M$ is the number of node degree allocations.

The coded bit sequence is bit-interleaved, and segmented into $\ell_{\text {map }}$-bit segments, and then each segment is mapped on to one of the $2^{m}$ constellation points for modulation. The complex-valued signal modulated according to the mapping rule is finally transmitted to the wireless channel. Since $\ell_{\text {map }}>m$ with $E M$, more than one label having different bit patterns in the segment are mapped on to each constellation point. However, there are many possible combinations of the bit patterns, hence determining of the optimal labeling pattern plays the key role to achieve limit-approaching performance.

\subsection{Channel}

This article assumes frequency flat additive white Gaussian noise (AWGN) channel. If the channel exhibits frequency selectivity due to the multipath propagation, the receiver needs an equalizer to eliminate the inter-symbol interference. Combining the technique presented in this article with the turbo equalization framework $[18,19]$ is rather straightforward. It is assumed that transmission chain is properly normalized so that the received $\mathrm{SNR}=1 / \sigma_{n}^{2}$; with this normalization, we can properly delete the channel complex gain term from the mathematical expression of the channel. The discrete time description of the received signal $y(k)$ is then expressed by

$$
y(k)=x(k)+n(k),
$$

where, with $k$ being the symbol timing index. $x$ is the transmitted modulated signal with unit power and expressed as

$$
x=\psi(s),
$$

where $s=\left[b_{1} b_{2} \ldots b_{\ell_{\text {map }}}\right]$ is labeling pattern and $\psi(\cdot)$ is the mapping function as indicated in Figure 1. $n$ is zero mean complex AWGN component with variance $\sigma_{n}^{2}$ (i.e., $\left\langle|x(k)|^{2}\right\rangle=1,\langle n(k)\rangle=0$ and $\left.\left\langle|n(k)|^{2}\right\rangle=\sigma_{n}^{2}\right)$ for $\forall k$.

\subsection{Receiver}

At the receiver side, the iterative processing is invoked, where extrinsic information is exchanged between the demapper and decoder. Using received signal sample $y$ $(k)$ and the a priori LLR fed back from the decoder, the demapper calculates the extrinsic LLR, $L_{e, D e m}\left[b_{v}(x(k))\right]$,

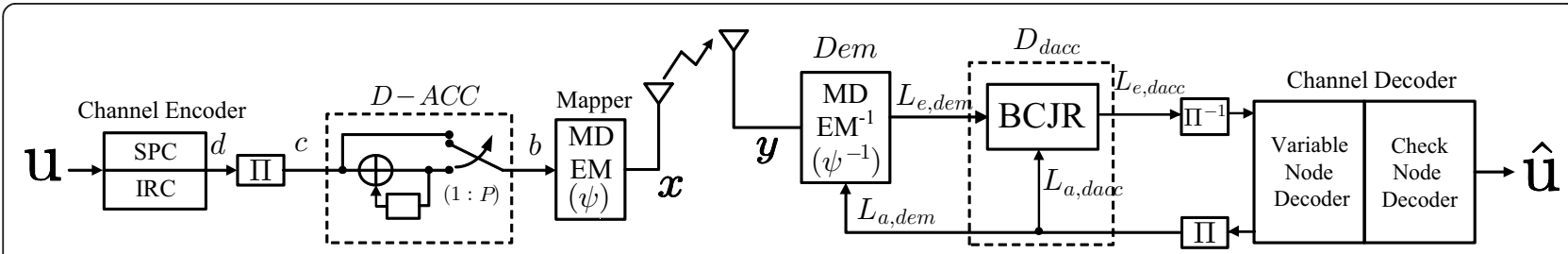

Figure 1 Proposed system structure. 
of the $v$ th bit in the labeling vector in the symbol $x(k)=$ $\psi(s(k))$ transmitted at the $k$ th symbol timing by

$$
L_{e, D e m}\left[b_{v}(x(k))\right]=\ln \frac{\sum_{s \in S_{o}} \exp \left\{-\frac{|\gamma(k)-\psi(s)|^{2}}{\sigma_{n}^{2}}\right\} \prod_{\rho=1, \rho \neq \nu}^{\ell_{\operatorname{map}}} \exp \left\{-b_{\rho}(s) L_{a, \text { Dem }}\left[b_{\rho}(s)\right]\right\}}{\sum_{s \in S_{1}} \exp \left\{-\frac{|\gamma(k)-\psi(s)|^{2}}{\sigma_{n}^{2}}\right\} \prod_{\rho=1, \rho \neq \nu}^{\ell_{\operatorname{mp}}} \exp \left\{-b_{\rho}(s) L_{a, \text { Dem }}\left[b_{\rho}(s)\right]\right\}}
$$

where $\mathrm{S}_{0}\left(\mathrm{~S}_{1}\right)$ indicates the set of the labeling pattern $s$ having the $v$ th bit being $0(1)$, and $L_{a, D e m}\left(b_{\rho}(s)\right)$ is the demapper's a priori LLR fed back from the decoder corresponding to the $\rho$ th position in the labeling pattern $s$.

Decoding takes place segment-wise where, because of the irregular code structure, the variable node degrees $d_{v_{i}}$ have different values segment-by-segment. Structure of the decoder as well as decoding algorithm is detailed in the previous publications, e.g., in $[13,14,20]$. Therefore, only summary of the algorithm is provided in this article.

The $d_{v_{i}}$ bits constituting one segment, output from the de-interleaver are connected to a variable node, and $d_{c}$ variable nodes are further connected to a check node; those demapper output bits in one segment, connected to the same variable node decoder, are not overlapping with other segments. Therefore, no iterations in the decoder are required $[13,14,20]$. The extrinsic LLR update for a bit at the check node is exactly the same as the check node operation in the LDPC codes, as

$$
\begin{aligned}
& L_{e, \mathrm{Cnd}, v}=\sum_{\kappa=1, \kappa \neq \nu}^{d_{c}} \boxplus L_{a, \mathrm{Dec}, \kappa} \\
& =2 \arctan \left(\prod_{\kappa=1, \kappa \neq \nu}^{d_{c}} \tanh \left(\frac{L_{a, \mathrm{Dec}, \kappa}}{2}\right)\right)
\end{aligned}
$$

where $\Sigma \boxplus$ represents the box-sum operator [5]. $L_{a}$, Dec, $\kappa=L_{e, D e m, \kappa}$ is the a priori LLR provided by the $\kappa$ th variable node. $L_{e, C n d, v}$ is the extrinsic LLR fed back to the $v$ th variable node, where it is further combined with $\left(d_{v_{i}}-1\right)$ a priori LLRs forwarded from the demapper via the deinterleaver, as

$$
L_{e, \text { Dec }, v}=L_{e, \text { Cnd }, v}+\sum_{\omega=1, \omega \neq v}^{d_{v_{i}}} L_{a, \text { Dec }, \omega}
$$

This process is performed for the other variable nodes in the same segment having the variable node degree $d_{v_{i}}$, and also for all the other segments independently in the same transmission block. Finally, the updated extrinsic LLRs obtained at the each variable node are interleaved, and fed back to the demapper. For the final bitwise decision, a posteriori LLR output from the decoder is used.

\subsection{DSI-BICM-ID-EM}

Reference [20] proposes a combined use of D-ACC with SI-BICM-ID-EM (DSI-BICM-ID-EM). ${ }^{\text {t }}$ The purpose of introducing $\mathrm{D}$-ACC is to lift the right-most point of demapper EXIT curve up to reach the (1.0,1.0) MI point so that the BER floor with SI-BICM-ID-EM can be eliminated. In this system structure, D-ACC is placed between the interleaver and mapper as shown in Figure 1 of [20]. The coded bit sequence is bit-interleaved, and input to the D-ACC with doping ratio of $(1: P)$. To keep the D-ACC's code rate equal to one, the interleaver's output is replaced by a D-ACC-coded bit at every $P$ th bit.

\subsection{MDSI-BICM-ID-EM}

This article also applies the idea of mixing modulation symbols of EM and standard Gray mapping at a certain ratio, original idea of which was first introduced in $[17,21]$. This technique is referred to as MD. Since the demapper EXIT function with Gray mapping is completely flat for 4-QAM, its left-most point has obviously higher MI than with EM mapping. The left-most point of demapper's EXIT curve is lifted up with MD and the amount depends on the mixing ratio. Figure 2 shows a block diagram of the MD system. The spectrum efficiency of the system then becomes $(D \cdot m+(1-D)$. $\left.\ell_{\text {map }}\right) \cdot R$ bits/symbol, where $D$ and $(1-D)$ are the ratios of the symbols with doped (Gray) and EM, respectively, in one transmission frame.

\section{EXIT analysis}

For the decoder of D-ACC, denoted as $D_{\text {dacc }}$, the BahlCocke-Jelinek-Raviv (BCJR) algorithm is performed at the receiver. Figure 3 show the EXIT curves for DemDdacc for $P=\{50,100,150,200\}$ : Figure 3 a depicts the entire portion of the curves, and Figure $3 \mathrm{~b}$ zooms up their right-most parts. It is observed that all demapper curves can achieve the right-most point close enough to the $(1.0,1.0)$ MI point. Furthermore, the $P$ value affects the shape of the demapper curve. It can be observed in Figure $3 \mathrm{~b}$ that the larger the $P$ value, the sharper the decay of the curve around the $(1.0,1.0)$ MI point.

Since detailed investigation for the effect of EM on the shape and the demap-per's EXIT function is provided in the previous publications, e.g., [20], they are not provided in this article. For those readers who are interested in this issue can refer $[7,13,20]$.

With the Gaussian assumption for the LLR distribution, the EXIT function of the repetition code decoder is given by

$$
I_{e, v}=J\left(\sqrt{\left(d_{v}-1\right) \cdot J^{-1}\left(I_{a, v}\right)^{2}}\right) .
$$




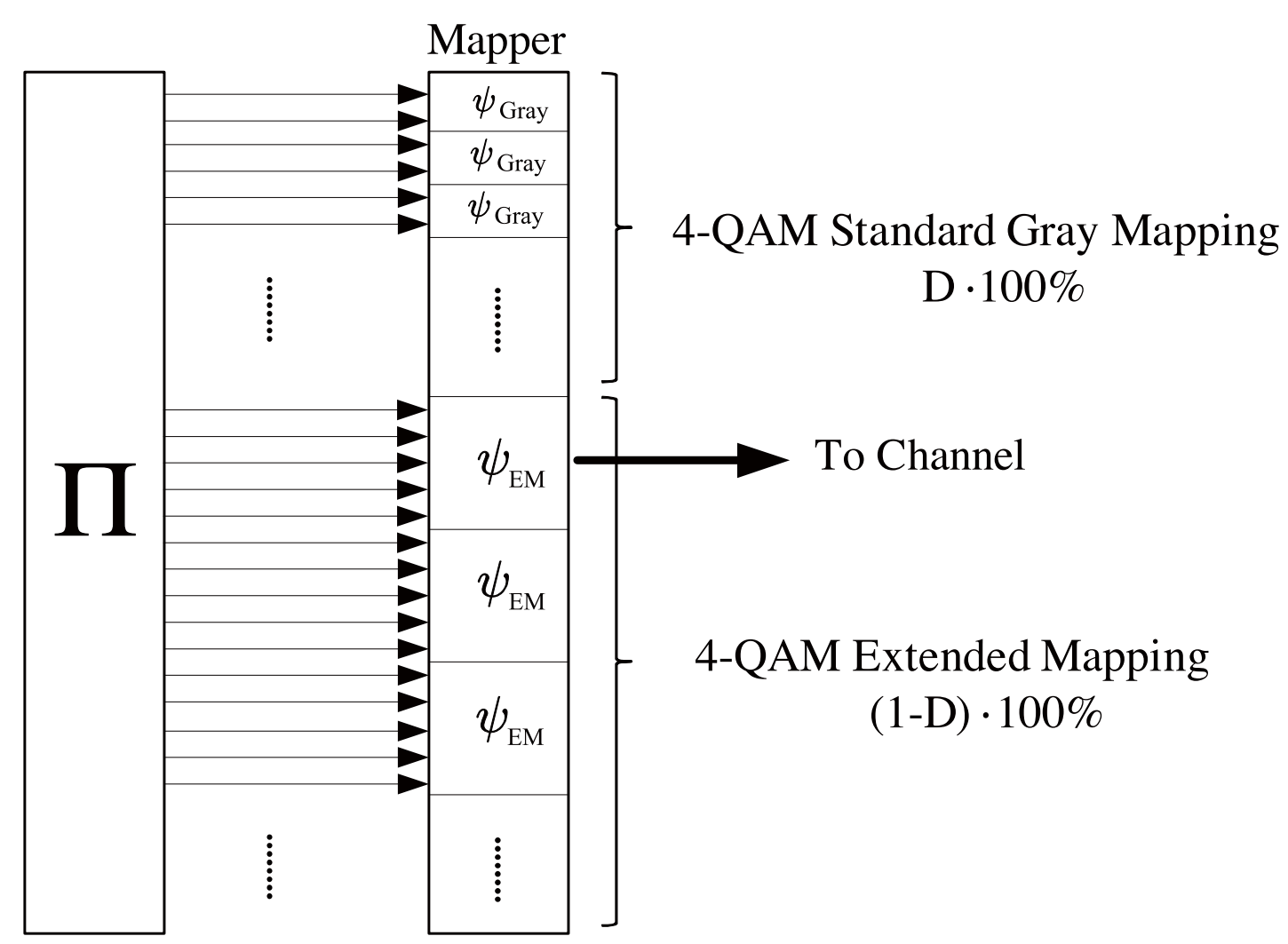

Figure 2 Single parity check irregular repetition coded BICM-ID with EM

where $I_{a, v}$ is a priori $\mathrm{MI}$ and $I_{e, v}$ is its output extrinsic MI. J(.) and $J^{-1}(\cdot)$ are the functions that convert the squareroot variance of LLR to its corresponding MI, and its inverse function, respectively [3]. Obviously, (9) is corresponding to the second term of the right hand side of (8) for LLR update, with which $I_{a, v}=I_{e, d e m}$, where $I_{e, d e m}$ is the demapper output extrinsic MI. The EXIT function of the SPC decoder can be approximated by [22]

$$
I_{e, \text { Cnd }}=1-J\left(\sqrt{d_{c}-1} \cdot J^{-1}\left(1-I_{a, \text { cnd }}\right)\right),
$$

where

$$
I_{a, \text { cnd }}=J\left(\sqrt{d_{v} \cdot J^{-1}\left(I_{a, \mathrm{Dec}}\right)^{2}}\right) .
$$

The EXIT function of the whole decoder comprised of the variable and check node decoders can be calculated by combining (10) and (11) [5], as

$$
I_{e, \mathrm{Dec}}=J\left(\sqrt{\left(d_{v}-1\right) \cdot J^{-1}\left(I_{a, \mathrm{Dec}}\right)^{2}+J^{-1}\left(I_{e, \mathrm{Cnd}}\right)^{2}}\right),
$$

with $I_{a, \text { Dec }}=I_{e, \text { dem }}$. Since the SI-BICM-ID-EM uses irregular structure of the repetition code, its EXIT function depends on the degree distribution $a_{i}$ of the variable node $d_{v_{i}}$. Our previous publication [13] showed that the EXIT function of the whole decoder with the proposed structure can be obtained by weighting the segment-wise EXIT functions, as

$$
I_{e, \text { Dec }}=\frac{\sum_{i} a_{i} \cdot d_{v_{i}} \cdot J\left(\sqrt{\left(d_{v_{i}}-1\right) \cdot J^{-1}\left(I_{a, \text { Dec }}\right)^{2}+J^{-1}\left(I_{e, \text { Cnd }}\right)^{2}}\right)}{\sum_{i} a_{i} \cdot d_{v_{i}}},
$$

Hence, it is found that the key of achieving the best matching between the demapper and the decoder EXIT curves is to jointly optimize the labeling pattern and the variable node degree distribution $a_{i}$.

\section{Framework for EBSA-based DSI-BICM-ID-EM design}

4.1 Optimal node degree allocation using LP

In [14], we showed that the optimal node degree allocations problem can be formulated as

$$
\begin{aligned}
& \text { Minimize } \sum_{i=1}^{M} a_{i} d_{v_{i}} \\
& \text { Subject to } \\
& \begin{array}{l}
\sum_{i=1}^{M} a_{i} \cdot d_{v_{i}}\left(-J\left(\sqrt{\left(d_{v_{i}}-1\right) \cdot J^{-1}\left(I_{e, D e m, w}\right)^{2}+J^{-1}\left(I_{e, C n d, w}\right)^{2}}\right)+I_{a, D e m, w}+\varepsilon_{w}\right) \\
\quad \leq 0(\text { for } 1 \leq w \leq N)
\end{array} \\
& \text { and } \sum_{i=1}^{M} a_{i}=1
\end{aligned}
$$




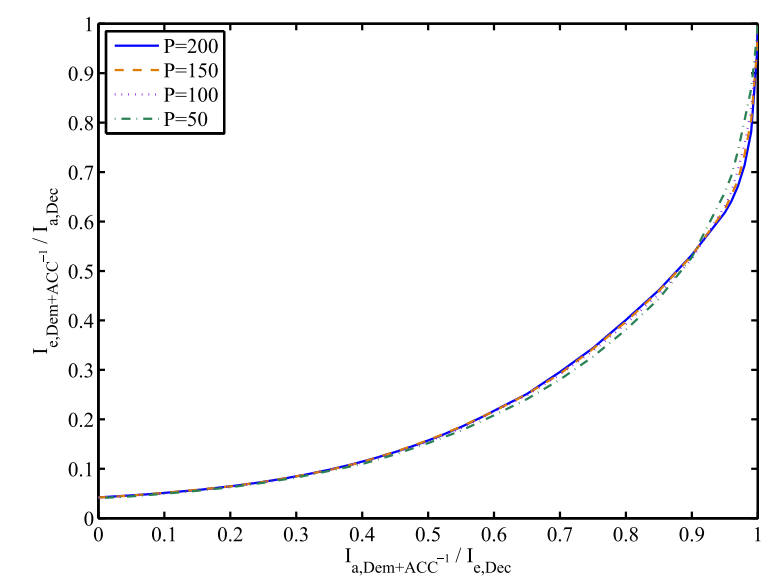

(a) $\mathrm{SNR}=0.8 \mathrm{~dB}$

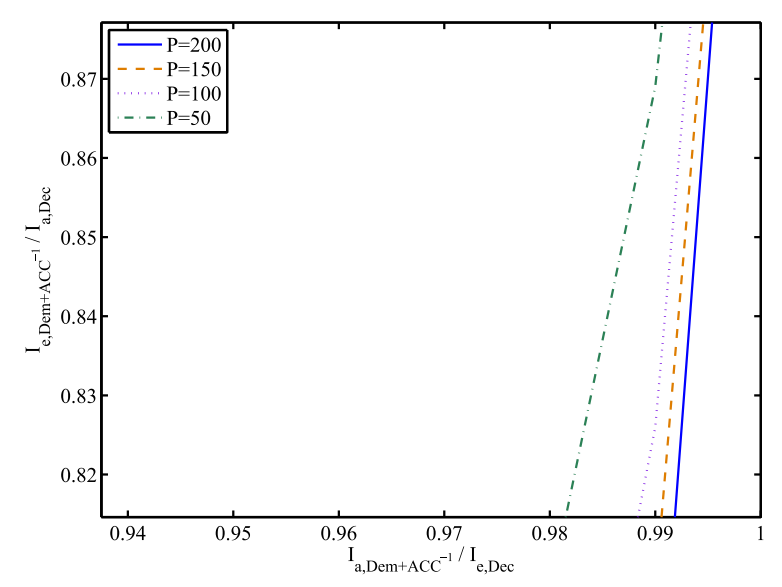

(b) $\mathrm{SNR}=3.1 \mathrm{~dB}$

Figure 3 EXIT curves of D-ACC-aided demapper

where the optimization variables are $a_{i},(i=1, \ldots, M)$ and $\epsilon_{w}$ denotes the pre-defined horizontal gap width between the demapper and the decoder EXIT curves and $N$ is the number of the MI constellation points as shown in Figure 4.

More details are given in Appendix 1. Furthermore, to find the optimal check node degree $d_{c}$, this article proposes a brute-force search (all possible value search), ${ }^{\mathrm{c}}$ as summarized in Algorithm 1.

Algorithm 1 Optimal degree allocation algorithm

Initialize $d_{v_{i}}$ and $a_{i}$ values.

for $d_{c}=2$ to $\max d_{c}$ do

Perform LP for Equation (14) with fixed $d_{c}$ and obtain optimal distribution $a_{i}$ for each $d_{v_{i}}$.

Calculate code rate $R$ using $d_{c}, d_{v}$ and $a_{i}$. end for

Find $d_{c_{\mathrm{opt}}}$ and $\mathbf{a}_{\mathrm{opt}}$ achieving $R \rightarrow \max$. return $d_{c_{\mathrm{opt}}}$ and $\mathbf{a}_{\mathrm{opt}}$

\subsection{EBSA framework}

In [12], Yang et al. introduce the idea of Adaptive BSA (ABSA) which takes into account the costs at multiple $a$ priori information points. The gap width between the demapper and the decoder EXIT curves is also taken into account, given the decoder EXIT curve. ABSA then obtains the optimal doping ratio in conjunction with determining the optimal labeling pattern. Hence, opening of the convergence tunnel until the $(1.0,1.0) \mathrm{MI}$ point is guaranteed with this technique. However, ABSA does not change the code parameters in optimization process, and therefore, optimality is only on given code-basis.

In this section, a novel technique EBSA is introduced. EBSA aims jointly to optimize labeling patterns, doping ratio, and code parameters using LP described in Section 4.1. Hence, EBSA achieves close matching between the demapper and the decoder curves, while it guarantees the opening of the convergence tunnel until the $(1.0,1.0)$ MI point. In a graphical expression, as presented in Figure 5, EBSA takes into account the horizontal and vertical gap widths at the multiple pre-defined a priori MI points, which is also effective in making a reasonable compromise between performance and complexity due to the turbo iterations.

Since both the ABSA and EBSA algorithms, in common, are based on the BSA, as well as the same cost definition, BSA and the cost are summarized in Appendices 2 and 3, respectively, for the completeness of the article. This article's proposed EBSA algorithm is summarized in Algorithm 2. It should be noticed that the processes for determining the optimal doping ratio, the $d_{c}$ value, and the LP based code parameter optimization are all included in a single repeat-until loop. This indicates that the code parameters are also changed in the EBSA framework.

It should be further noticed that the horizontal and vertical gap widths evaluation, as descriptively summarized in Figure 5, is included in the repeat-until loop. With the EBSA framework, the labeling pattern used in the LP-based degree allocation optimization for DSIBICM-ID-EM are obtained by lowering the cost of $Z_{\ell_{\text {map }}-1}$ (at right-most MI point corresponding to the case with full a priori information) as much as possible, while still keeping the vertical gap smaller than the predefined value $\delta_{w}$. Hence, other costs $Z_{0}, \ldots, Z_{\ell_{\text {map }}-2}$ are ignored in the LP based optimization.

\section{Numerical results}

\subsection{Convergence property analysis}

The EBSA optimization technique is a design framework for BICM-ID, and therefore applicable not only to 


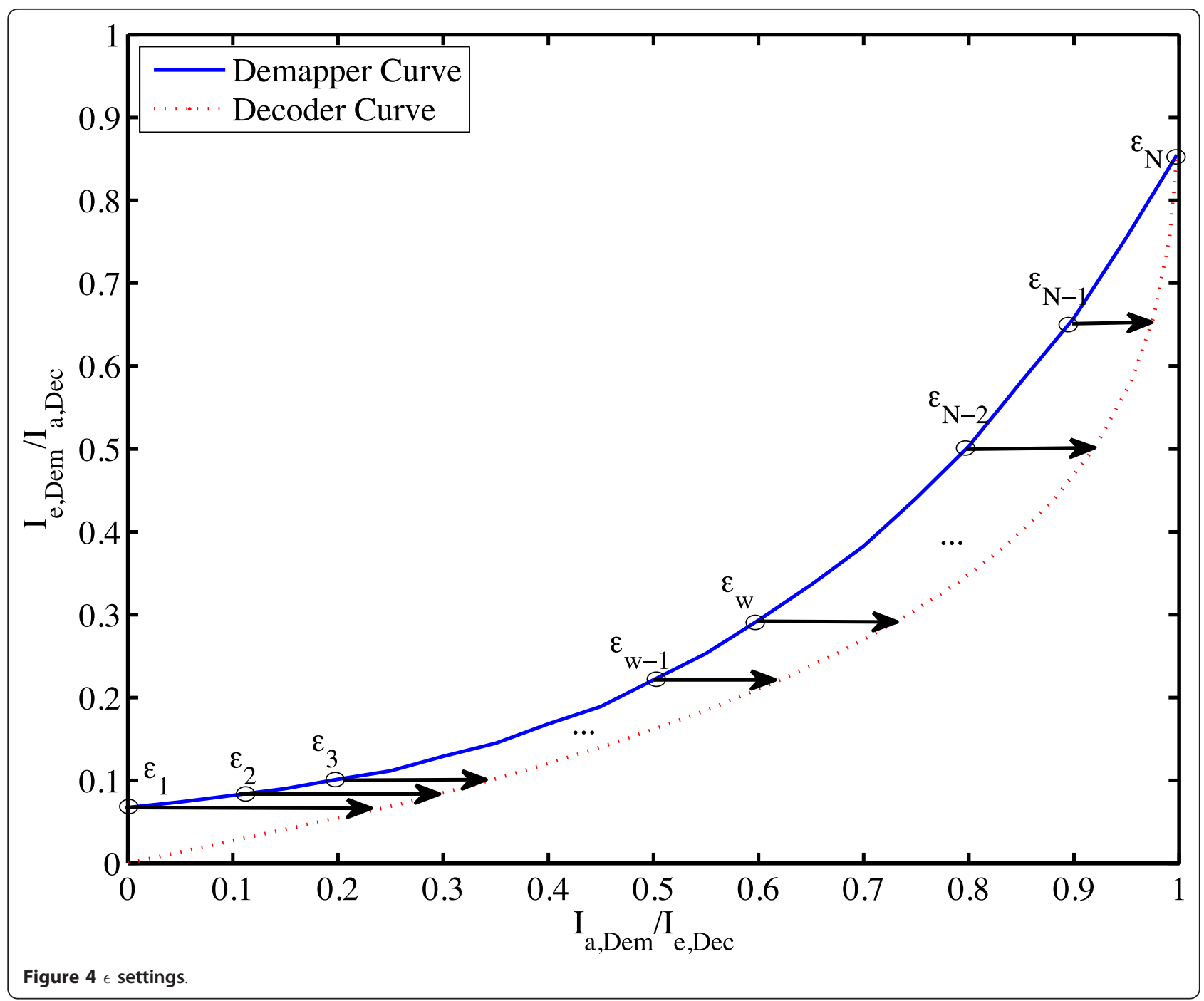

MDSI-BICM-ID-EM, but also to other structures, as described in Endnote " $a$ " in Section 1. To demonstrate the performances superiority with the optimization techniques described in this article, EXIT curves were calculated for several designs described in the previous sections, aiming at the turbo cliff to happen at $\mathrm{SNR}=$ $0.8 \mathrm{~dB}$ and $3.1 \mathrm{~dB}$, as examples.

\subsubsection{SI-BICM-ID-EM with node degree optimization using} LP

Tables 1 and 2 show the node degree allocations before and after performing LP for $\ell_{\text {map }}=5$ and $\mathrm{SNR}=0.8 \mathrm{~dB}$, where the simplex algorithm was used as a tool for LP. Table 3 shows the initial values of $\epsilon$ for the optimization. The expected

Algorithm 2 EXIT-constrained binary switching algorithm

$($ EBSA $) \lambda=\left[\begin{array}{ll}\lambda_{0} \lambda_{1} \cdots \lambda_{\ell_{\text {map }}-2} \lambda_{\ell_{\text {map }}-1}\end{array}\right]=\left[\begin{array}{lllll}0 & 0 & \cdots & 0 & 1\end{array}\right]$
Initialization: Generate labeling pattern $s$ randomly as well as degree distribution $d_{\nu}$ empirically

for $P=2$ and $d_{c}=2$ to $\max P$ and $\max d_{c}$, respectively do

\section{repeat}

Draw demapper EXIT curve based on the given labeling pattern $s$ obtained as the result of the latest iteration

$$
\text { for } i=1 \text { to } N_{\max }{ }^{\mathrm{d}} \text { do }
$$

$$
\text { Conduct BSA with } Z_{\ell_{\text {map }}-1}=\bar{Z} \text { and }
$$

$Z_{\ell_{\text {map }}-1}^{h}=\bar{Z}^{h}\left(h=0, \ldots 2^{\ell_{\text {map }}-1}\right)$

\section{end for}

Draw the demapper's EXIT curve using the obtained labeling pattern.

Draw the decoder's EXIT curve using degree distribution using LP for Equation (14) with the obtained labeling pattern. 


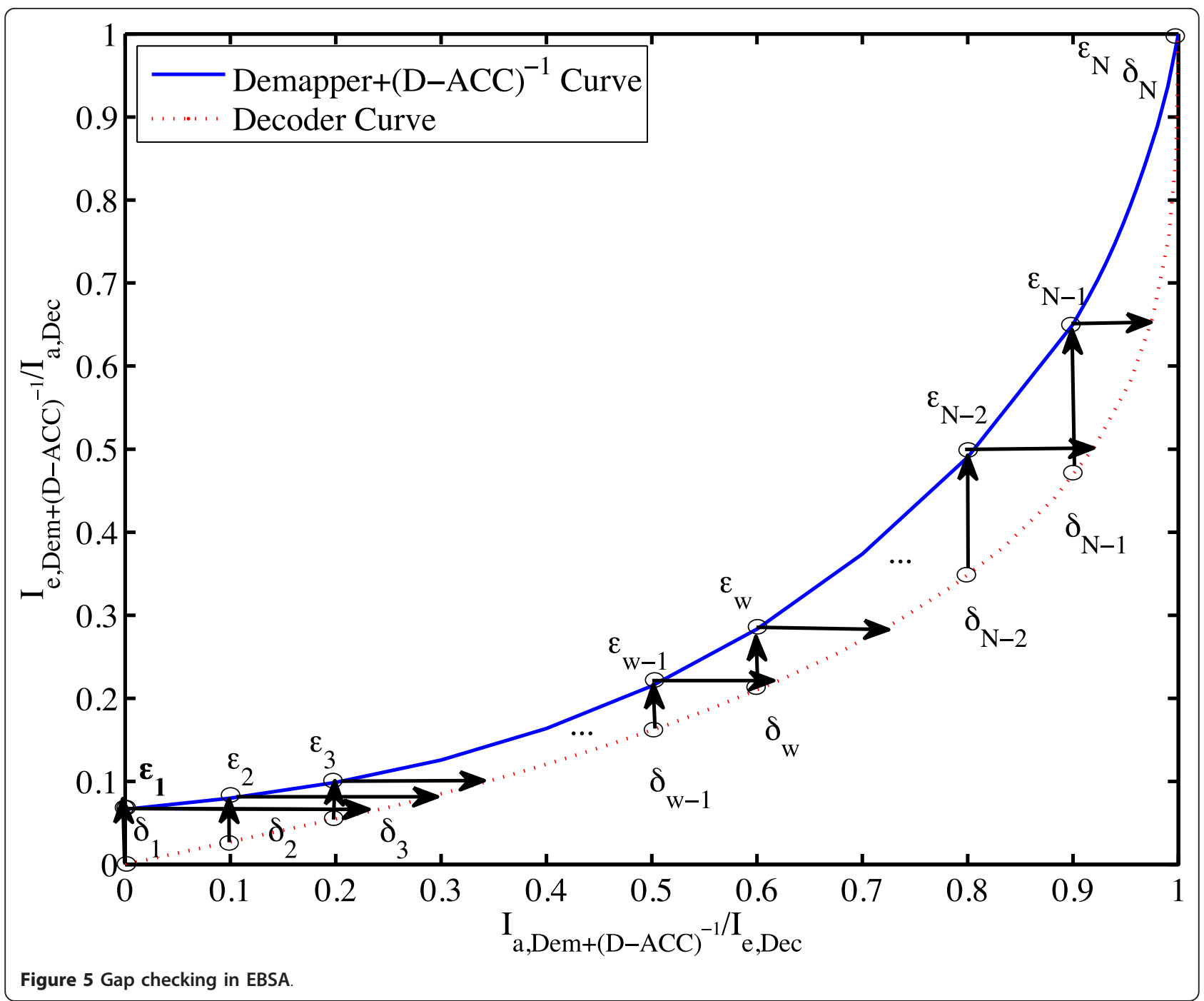

if *The vertical gap $\delta$ in the range of $M I\left(Z_{q} \pm\right.$ $\left.\Delta Z_{q}\right)^{\mathrm{e}}, 0 \leq q \leq \ell_{\text {map }}-1$, around $Z_{q}$ as shown in Figure 6 is larger than desired value $\delta_{q}$ then

$$
\lambda_{q}=\lambda_{q}-1
$$

\section{end if}

Select the labeling pattern and decoder node distribution that has minimum gap.

until Gap between the demapper and decoder EXIT curve becomes smaller than the threshold for each MI points tested.

end for

Select the optimal parameter set that minimizes the gap

Table 1 Initial degree distributions

\begin{tabular}{ccccccccccc}
\hline$d_{v}$ & 1 & 2 & 3 & 4 & $\ldots$ & 6 & 7 & 8 & 9 & 30 \\
\hline \multirow{2}{*}{ a } & $\frac{1}{30}$ & $\frac{1}{30}$ & $\frac{1}{30}$ & $\frac{1}{30}$ & $\ldots$ & $\frac{1}{30}$ & $\frac{1}{30}$ & $\frac{1}{30}$ & $\frac{1}{30}$ & $\frac{1}{30}$ \\
\hline
\end{tabular}

intersection point is set at $I_{a, D e m}=0.9999$. With the same initial degree allocations and $\epsilon$ settings, EBSA was performed for SNR $=3.1 \mathrm{~dB}$. Figure 7 show with and without optimization the EXIT curves for SNR $=0.8$ $\mathrm{dB}$ and $3.1 \mathrm{~dB}$, respectively, for SI-BICM-ID-EM. In the case without degree allocations optimization, the empirically obtained distribution shown in $[13,15]$ were used, which are indicated by (i) and (iii) in the figure for SNR $=0.8 \mathrm{~dB}$ and $3.1 \mathrm{~dB}$, respectively.

It is found by carefully looking at the right-most part of the curves that the intersection point of the (ii)-(iv) decoder curves and the demapper curve indicated by $\left({ }^{*}\right)$ in the figure are found to be slightly closer to the extrinsic $\mathrm{MI}=1.0$ than the empirically designed case.

Table 2 Optimized degree distributions

\begin{tabular}{ccccccccccc}
\hline $\boldsymbol{d}_{\boldsymbol{v}}$ & $\mathbf{1}$ & $\mathbf{2}$ & $\mathbf{3}$ & $\mathbf{4}$ & $\mathbf{5}$ & $\mathbf{6}$ & $\ldots$ & $\mathbf{2 3}$ & $\ldots$ & $\mathbf{3 0}$ \\
\hline$a$ & 0 & 0 & 0 & 0 & 0.9438 & 0.0419 & $\ldots$ & 0.0143 & $\ldots$ & 0
\end{tabular}


Table $3 \epsilon$ settings

\begin{tabular}{ccccccccccc}
\hline $\boldsymbol{w}$ & $\mathbf{1}$ & $\ldots$ & $\mathbf{6}$ & $\mathbf{7}$ & $\mathbf{8}$ & $\mathbf{9}$ & $\mathbf{1 0}$ & $\mathbf{1 1}$ & $\mathbf{\ldots}$ & $\mathbf{2 4}$ \\
\hline$\epsilon_{w}$ & 0.001 & $\ldots$ & 0.001 & 0.001 & 0.001 & 0.001 & 0.001 & 0.001 & $\ldots$ & 0 \\
\hline
\end{tabular}

However, the rate of the code obtained by LP is slightly lower than the rate of the code with empirically obtained degree allocation, and the intersection point of the two EXIT curves is still quite apart from the $(1.0,1.0)$ MI point. Therefore, LP alone can lower the BER floor, but cannot increase the spectrum efficiency in those cases.

\subsubsection{DSI-BICM-ID-EM with node degree optimization using}

\section{LP technique}

To eliminate the BER floor, we conducted a node degrees optimization for DSI-BICM-ID-EM aiming at better matching of the two EXIT curves. Figure 8a shows the EXIT curve with $D e m D_{\text {dacc }}$ for SNR $=0.8 \mathrm{~dB}$. In addition, the decoder EXIT curves are also drawn using the degree distribution obtained by using LP with the $\epsilon$ settings given in Table 3 . Note that the doping rate $P$ was determined empirically in this case. It can be observed from Figure 8a that the $D e m D_{\text {dacc }}$ and decoder EXIT curves closely match except the left-most region.

Similar result can be observed from Figure 8b, where optimization was performed for SNR $=3.1 \mathrm{~dB}$. Both in Figures $8 \mathrm{a}, \mathrm{b}$, the two EXIT curves intersect at a point very close to the $(1.0,1.0)$ MI point. Therefore, no BER floor (or, at least invisible in the BER value range shown in the figure) and higher spectrum efficiency compared to the empirically designed SI-BICM-ID-EM are expected.

\subsubsection{MDSI-BICM-ID-EM with EBSA}

Figure 9 shows the EXIT chart of DSI-BICM-ID-EM obtained by EBSA. Note that $\epsilon$ and $\delta$ settings shown in Tables 3 and 4 were used, respectively. It can be observed that as the result of EBSA, the shape of the $D e m D_{\text {dacc }}$ EXIT curve indicated by $(-)$ in Figure $8 \mathrm{a}$ is

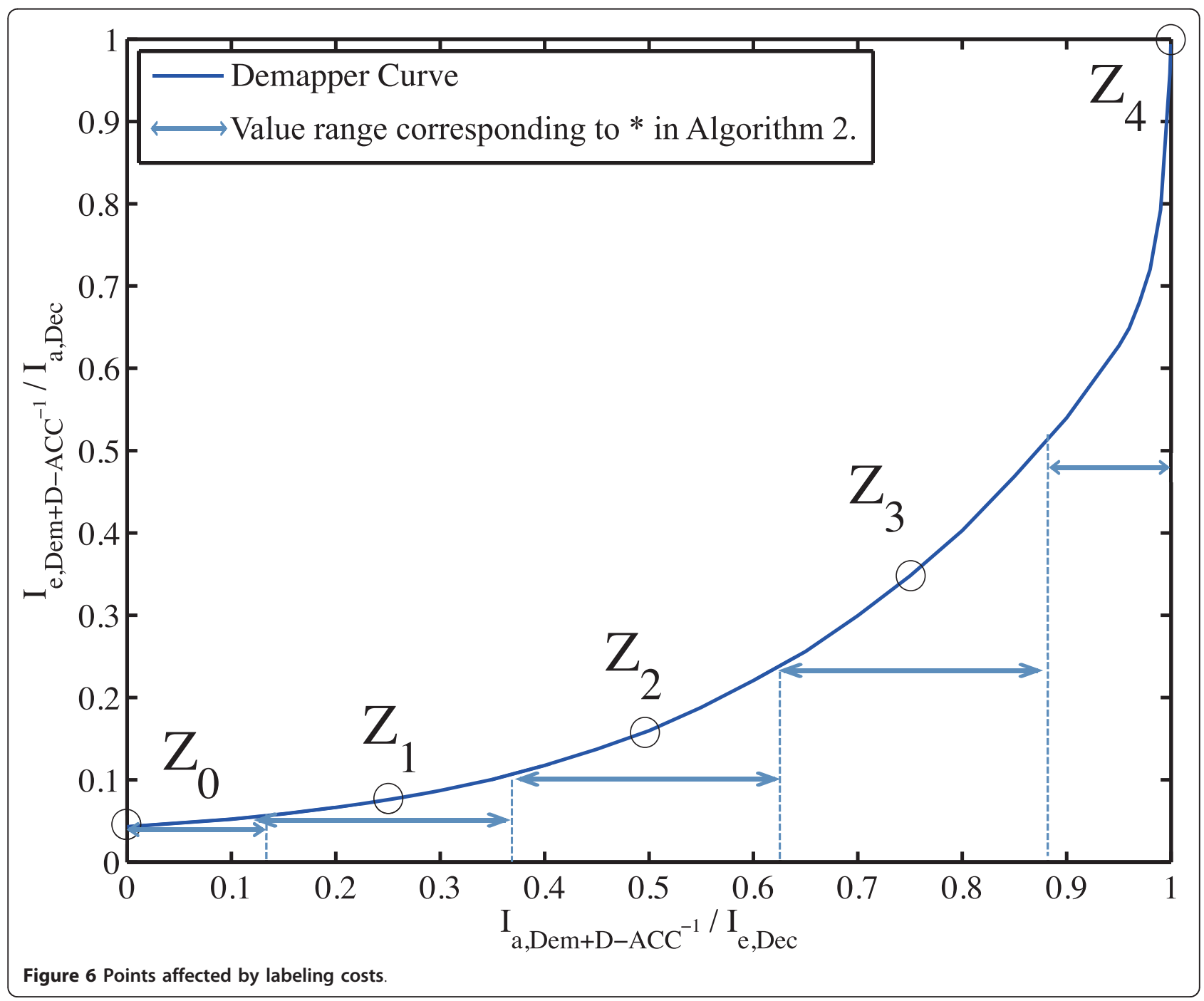




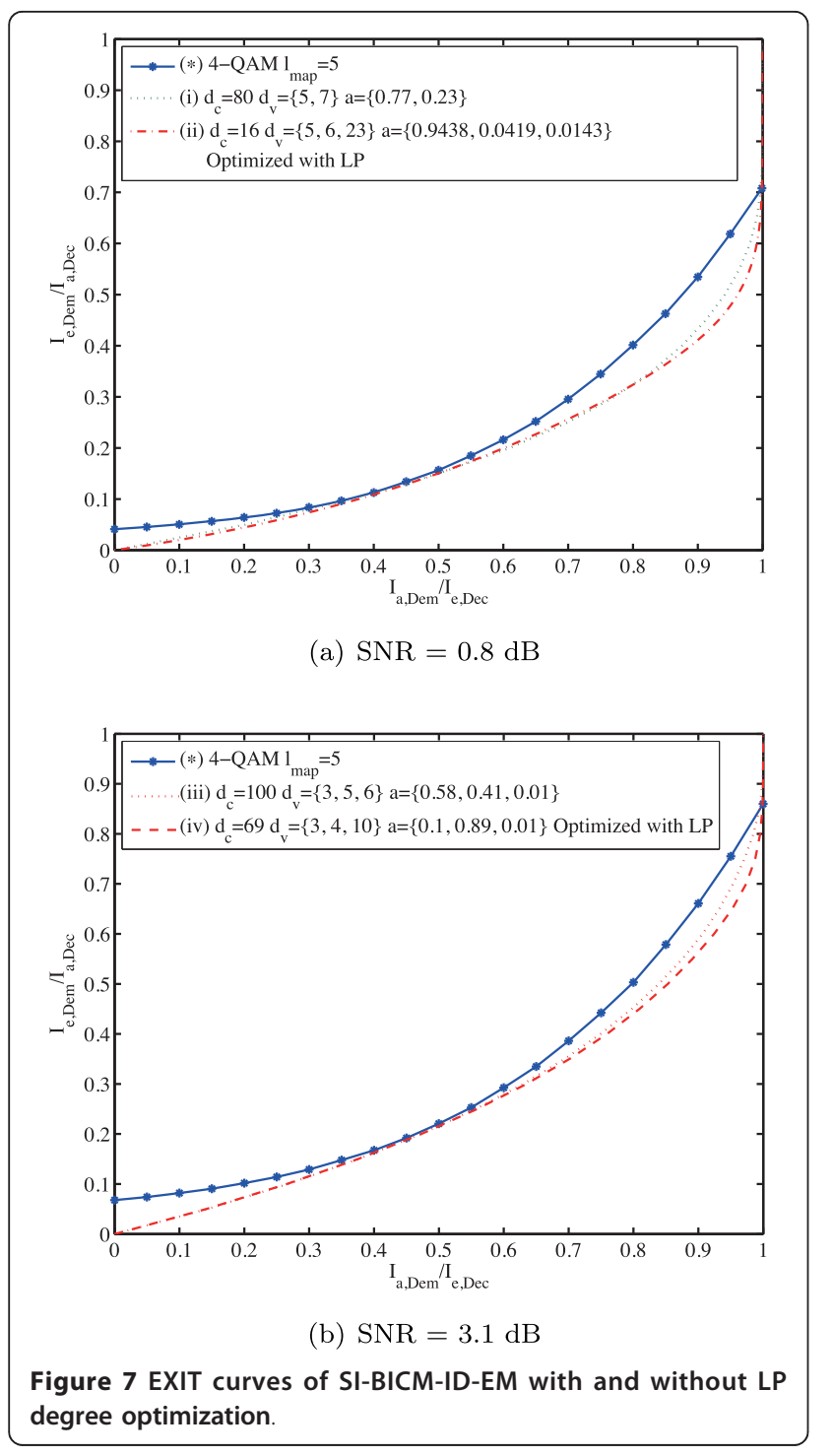

changed to that shown in Figure 9. Notice that the obtained demapper EXIT curve starts from the the $(0.0,0.0) \mathrm{MI}$ point. Therefore, no node degree distribution that can initiate the LLR exchange was found by LP for any given initial $\epsilon$ and $\delta$ settings. This is because EBSA aims to push down the $D e m D_{\text {dacc }}$ curve by allowing the gap width lower than $\delta_{w}$ for $1 \leq w \leq N$. Therefore, we apply EBSA to MDSI-BICM-ID-EM in order to slightly lift up the left-most point of the $D e m D_{\text {dacc }}$ EXIT curve. Figure 10 shows the EXIT curves, where we apply EBSA to MDSI-BICM-ID-EM with the MD ratio 0.012 and 0.01 for $\mathrm{SNR}=0.8 \mathrm{~dB}$ and $3.1 \mathrm{~dB}$, respectively. In the EXIT analysis in those cases, the labeling patterns shown in Figure 11, obtained as the result of EBSA, was used.

From Figure 10a, very close matching between the Dem $D_{\text {dacc }}$ and the decoder EXIT curves can be observed

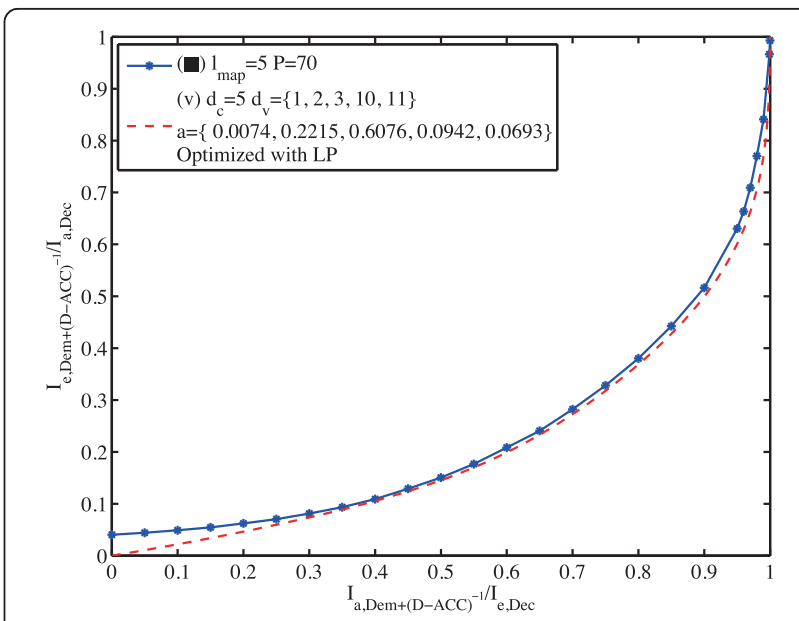

(a) $\mathrm{SNR}=0.8 \mathrm{~dB}$

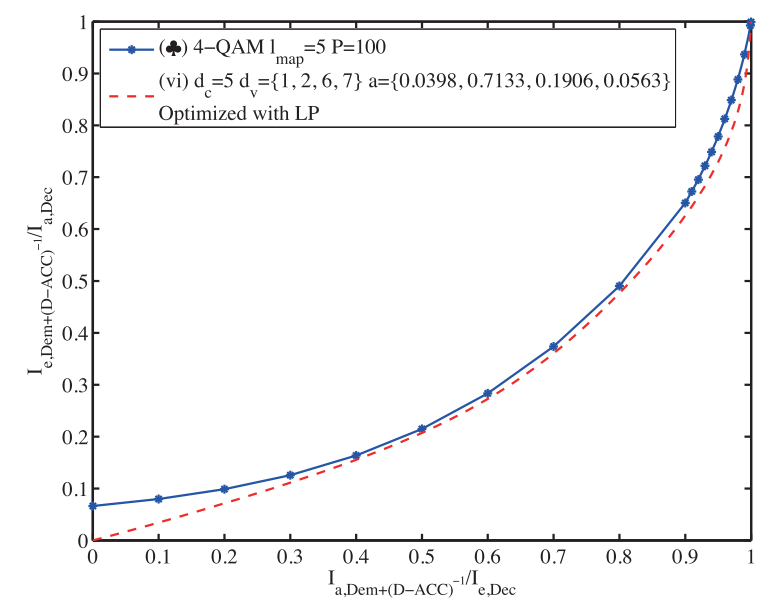

(b) $\mathrm{SNR}=3.1 \mathrm{~dB}$

Figure 8 EXIT curves of DSI-BICM-ID-EM with LP degree optimization.

from the starting point to the end. Moreover, now the Dem $D_{\text {dacc }}$ EXIT curve starts from $(0,0.0064)$ and thereby, LLR exchange can be initiated, and hence the trajectory can reach the target MI point close enough to the $(1.0,1.0)$ point. Similar characteristics can be observed in Figure 10b, where the optimization was performed for $\mathrm{SNR}=3.1 \mathrm{~dB}$.

\subsection{BER performances}

Figure 12 shows the BER performance using the proposed optimization techniques at the target $\mathrm{SNR} \approx 0.8$ $\mathrm{dB}$. It is found that for SNR $=0.8 \mathrm{~dB}$ when only node degree distribution optimization by LP was performed for SI-BICM-ID-EM (indexed by $\left(^{*}\right)$ and (ii) in the figure), lower bit error floor can be achieved compared to empirically designed case $\left(\left(^{*}\right)\right.$ and (i)) $[13,15]$. However, due to the decrease in the code rate, the Shannon limit 


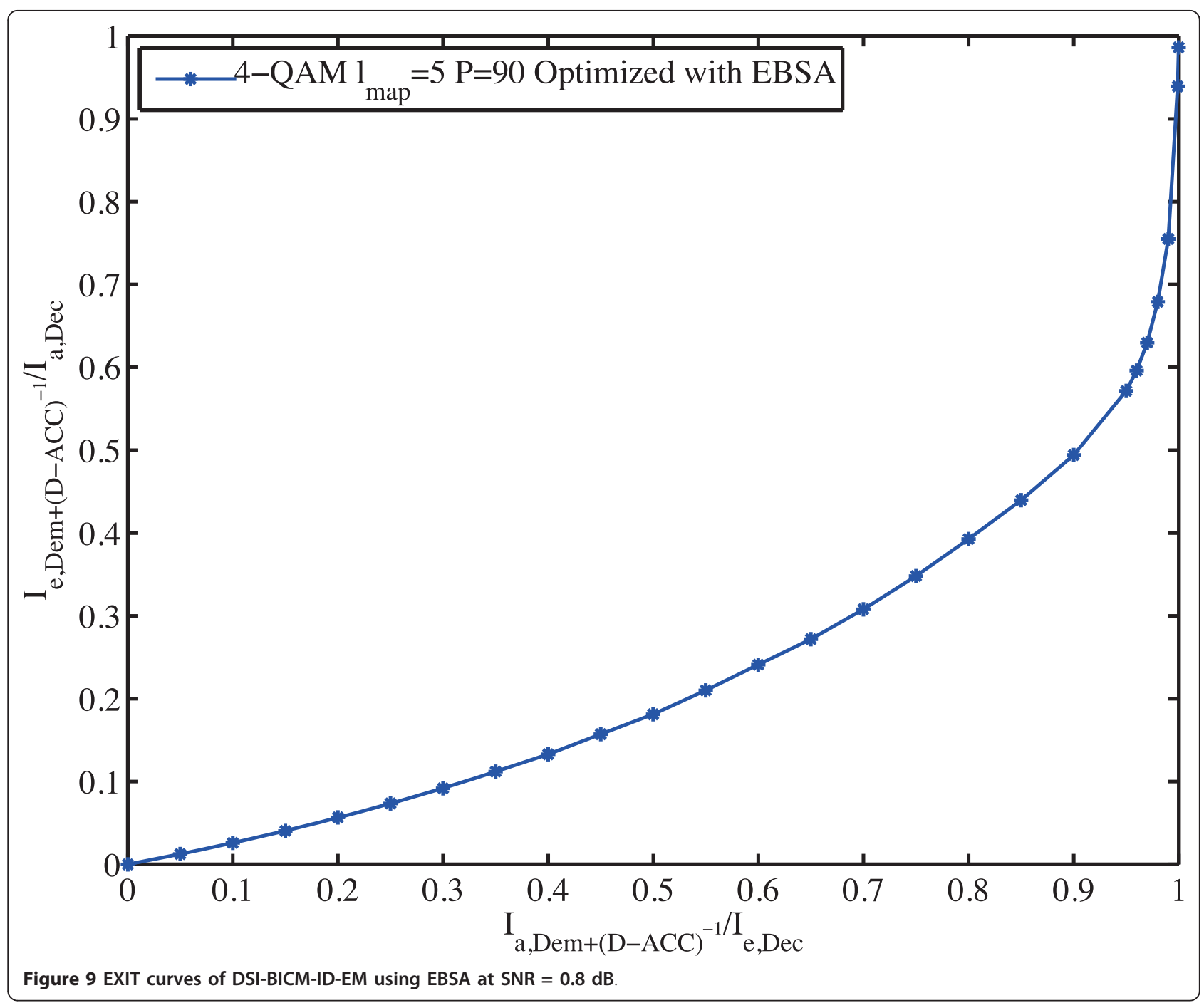

SNR becomes even lower than the empirically designed case $(1.5 \mathrm{~dB}$ away from the limit with $(*)$ and (i), while $1.6 \mathrm{~dB}$ away with (*) and (ii)). The curve indexed by $(*)$ and (v) shows the BER performance, where node degrees optimization was performed to DSI-BICM-IDEM. With this technique, turbo cliff happens about 0.8 $\mathrm{dB}$ away from the Shannon limit (corresponding to the curve indicated by ( - ) and (v) in the figure.). Furthermore, it is found that, by using the D-ACC, BER floor can be completely eliminated (or, at least invisible in the BER value range shown in the figure).

The BER performance with MDSI-BICM-ID-EM are shown by the curve indicated by ( $\mathbf{v}$ ) and (vii), where EBSA and MD were utilized. The best result among

Table $4 \delta$ settings

\begin{tabular}{ccccccccccc}
\hline $\boldsymbol{w}$ & $\mathbf{1}$ & $\ldots$ & $\mathbf{6}$ & $\mathbf{7}$ & $\mathbf{8}$ & $\mathbf{9}$ & $\mathbf{1 0}$ & $\mathbf{1 1}$ & $\mathbf{\ldots}$ & $\mathbf{2 4}$ \\
\hline$\delta_{w}$ & 0.001 & $\ldots$ & 0.001 & 0.001 & 0.001 & 0.001 & 0.001 & 0.001 & $\ldots$ & 0 \\
\hline
\end{tabular}

those tested cases achieves the threshold SNR of around $0.5 \mathrm{~dB}$ away from the Shannon limit, for which parameters are shown below the figure caption. It should be noted that approximately 300 iterations were needed for the BER simulations when SNR is around the threshold. Similar performance can be observed with the BER curves yielding turbo cliff at around $3.1 \mathrm{~dB}$ as shown in Figure 13.

\section{Complexity assessment}

With $\ell_{\text {map }}=5$, there are 32 labeling patterns in total, where each of the sets $S_{0}$ and $S_{1}$ contains 16 patterns. Hence, the probabilities for the 16 patterns have to be summed up when calculating the numerator and the denominator of (5). Since the BCJR algorithm requires forward and backward processing and each state emits two branches corresponding to the systematic input being 0 and 1 , the computational complexity for the demapper having 16 labeling patterns both in the 


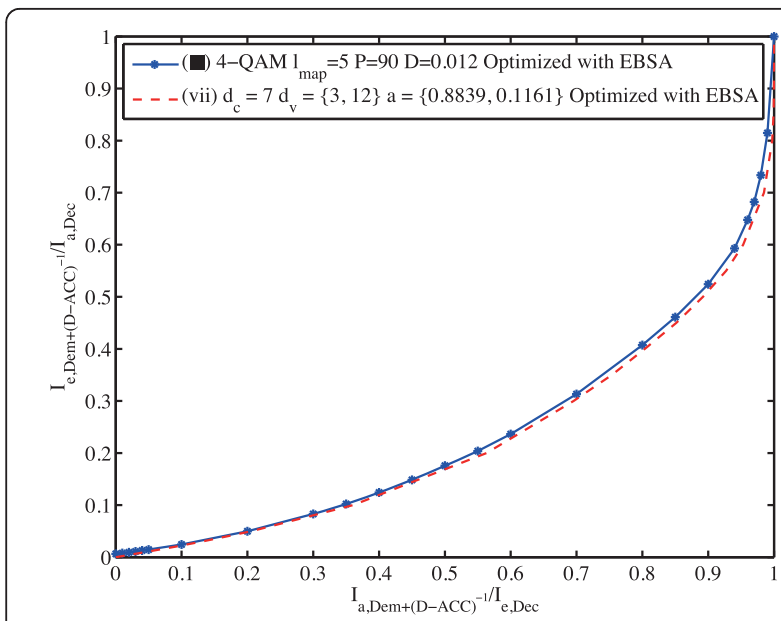

(a) $\mathrm{SNR}=0.8 \mathrm{~dB}$

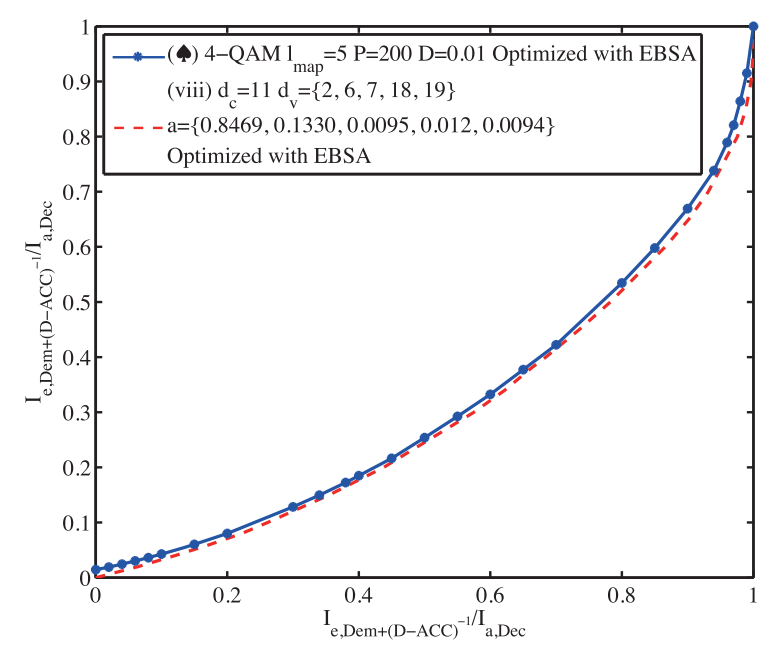

(b) $\mathrm{SNR}=3.1 \mathrm{~dB}$

Figure 10 Demapper's EXIT curve of MDSI-BICM-ID-EM using EBSA. numerator and the denominator is equivalent to the decoding complexity of memory- 3 convolutional code using the BCJR algorithm $\left(3=\log _{2}(8)=\log _{2}\left(\frac{16}{2}\right)\right)$. Furthermore, since Turbo code requires at least two constituency codes [1], the complexity estimated above is also roughly equivalent to that required by a Turbo code having two memory- 2 constituent convolutional codes $\left(2=\log _{2}(4)=\log _{2}\left(\frac{8}{2}\right)\right)$.

The decoding complexity for $D_{\text {dacc }}$ is negligible and so is the case of the decoding complexity for the SPC and IRC codes as well, because no iterations are needed in the decoder, as described in Section 2. Therefore, it can be concluded that with $\ell_{\text {map }}=5$ the computational complexity required for the proposed MDSI-BICM-IDEM technique is almost equivalent to that with a Turbo code having memory-2 constituent convolutional code. It should be emphasized here that the Turbo code proposed in [1] uses two memory-4 convolutional codes, which requires roughly 4 times as large complexity as that with the proposed technique with $\ell_{\text {map }}=5$. Nevertheless, the proposed technique can achieve better BER performance than the Turbo code presented in [1]. This is mainly because the EBSA algorithm jointly optimize the labeling patterns and degree allocations as a systematic framework. Numbers of the additions, multiplications, and divisions required for the demapping process are summarized in Table 5.

\section{Conclusions}

This article has proposed a design framework, EBSA, and applied it to our proposed BICM-ID techniques, SIBICM-ID-EM, DSI-BICM-ID-EM and MDSI-BICM-IDEM. Since EBSA takes into account the horizontal and vertical gap widths between the $\operatorname{Dem} D_{\text {dacc }}$ and decoder's EXIT curves at the pre-defined several MI points, it can determine the optimal labeling pattern for EM and degree allocations simultaneously. In fact, when EBSA is

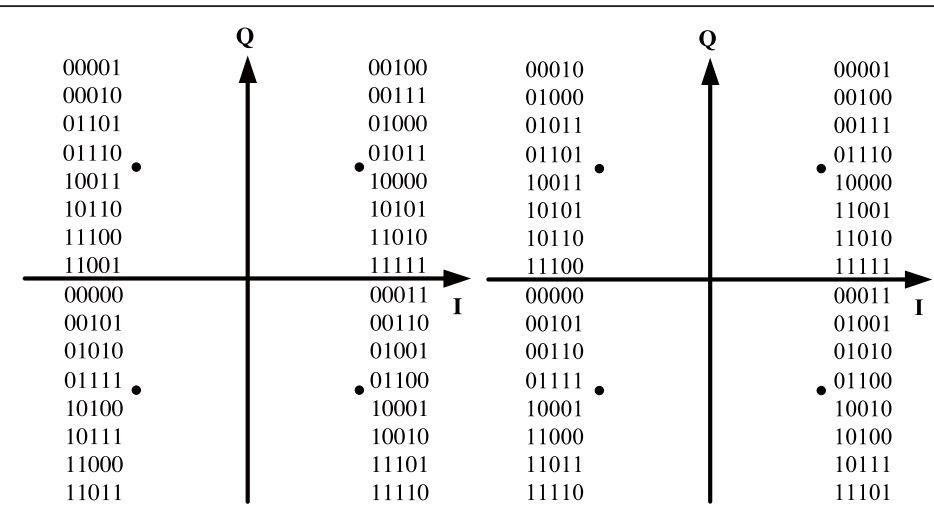

(a) $\mathrm{SNR}=0.8 \mathrm{~dB}$

(b) $\mathrm{SNR}=3.1 \mathrm{~dB}$

Figure 11 Obtained labeling pattern using EBSA. 


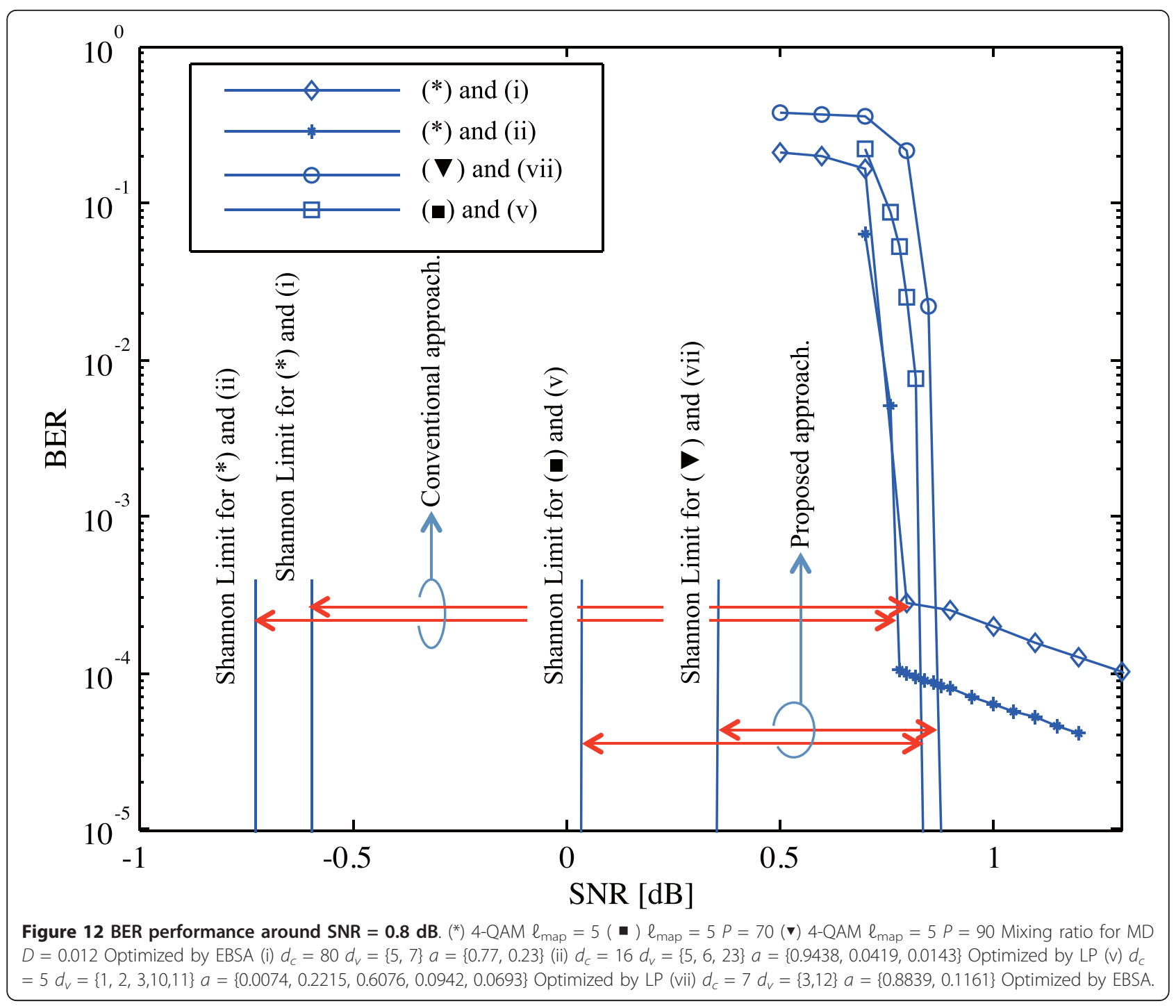

applied to DSI-BICM-ID-EM, two curves exactly match, and surprisingly the left-most point of the EXIT curve of $D e m D_{\text {dacc }}$ is determined to be the $(0.0,0.0)$ MI point, and hence LLR exchange can not be initiated. To avoid this situation, this article introduced the MD technique, by which the left-most point of the DemD $D_{\text {dacc }}$ EXIT curves can be lifted up slightly while the other part still exactly matched. As the result, very close-Shannon limit performance can be achieved without requiring heavy computational burden with $\ell_{\text {map }}=5$ EM 4-QAM; the complexity is almost the same level as a Turbo code with only memory- 2 constituency codes.

The following three issues have to be noted in concluding this article, since this special issue has two focal points, "Algorithm and Implementation Aspects":
- The proposed EBSA is applicable to BICM-ID techniques using other codes, so far as the degree allocation optimization can be performed using LP. LDPC-aided BICM-ID [23] and irregular convolutional code-aided BICM-ID [16] belong to this category. This is the reason why call EBSA "framework" rather than "technique".

- The trade-off between performance and complexity due to iterations can well be managed with EBSA by properly setting the horizontal and vertical gap parameters, $\epsilon$ and $\delta$, respectively, at several MI points. Even relatively large gap parameters are used so that not too many iterations are required, still arbitrary low BER can be achieved because the two curves reach a point close enough to the $(1.0,1.0) \mathrm{MI}$ point. 


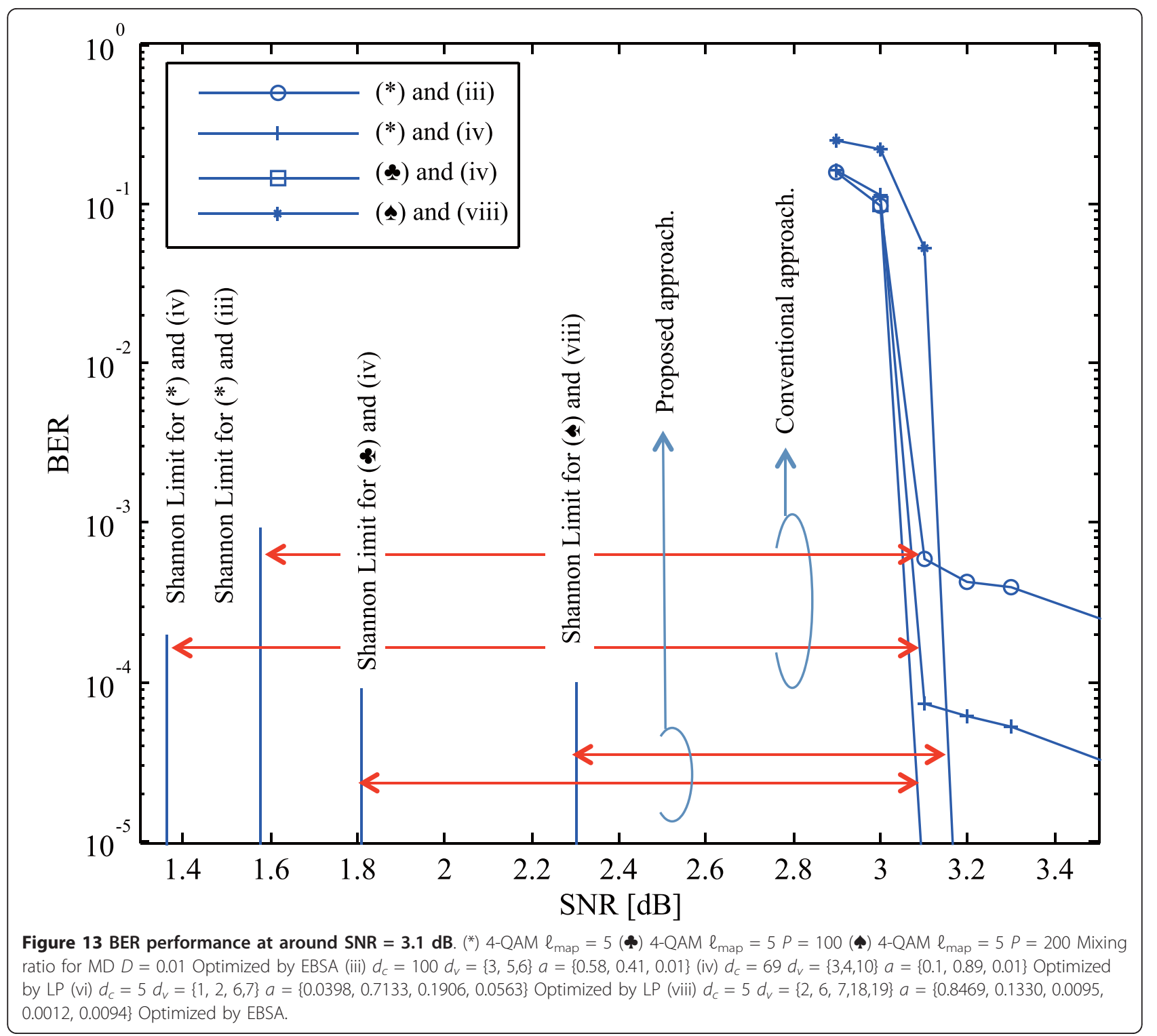

- Application of the EBSA framework to higher order modulation is left as future study.

\section{Appendix 1: Node degree optimization using LP}

The objectives of the node degree optimization can be defined as follows.

1. Code rate has to be lower than but as close to the capacity as possible.

2. Keep the convergence tunnel open between the demapper and decoder EXIT curve until the desired intersection point and the point should be as close to the $(1.0,1.0) \mathrm{MI}$ point as possible.

3. Total of node degrees distributions has to be always 1 .
Given $\ell_{\text {map }}$, the criterion can be written as

Find $a_{i}$ for each $d_{v_{i}}$

Such that Rate $=\left(d_{c}-1\right) /\left(d_{c} \sum_{i=1}^{M} a_{i} \cdot d_{v_{i}}\right) \rightarrow$ Maximized

$$
\begin{aligned}
& \text { Subject to } \sum_{i=1}^{M} a_{i}=1, \\
& \text { and } I_{e, \text { Dec }}>I_{a, \text { Dec }}=I_{e, \text { Dem }},
\end{aligned}
$$

where $I_{e \text {,Dec }}, I_{a, \text { Dec }}$ and $I_{e, \text { Dem }}$ denote the decoder extrinsic MI, the decoder a priori MI and the demapper extrinsic MI, respectively. Now, assume that the check node degree $d_{c}$ is a constant. Then, the optimality criteria of (15) is equivalent to 
Table 5 Calculation cost of 4-QAM EM

\begin{tabular}{cccc}
\hline & Addition/Subtraction & Multiplication & Division \\
\hline 4-QAM EM & $2^{\ell_{\text {map }}} \ell_{\text {map }}^{2}$ & $2 \ell_{\text {map }}^{2}-2$ & $3 \ell_{\text {map }}$ \\
\hline
\end{tabular}

Find $a_{i}$ for each $d_{v_{i}}$

Such that $\sum_{i=1}^{M} a_{i} \cdot d_{v_{i}} \rightarrow$ Minimized

Subject to $\quad \sum_{i=1}^{M} a_{i}=1$

and

$$
I_{e, \mathrm{Dec}}>I_{a, \mathrm{Dec}}=I_{e, \mathrm{Dem}}
$$

where the, index $w$ is introduced, representing the pre-defined MI constraint points, and also another parameter $\epsilon_{w}$ representing the acceptable gap between $I_{e, \text { Dec }}$ and $I_{a, \text { Dec }}$, such that

$$
I_{e, \text { Dec }, w}-I_{a, \text { Dec }, w} \geq \varepsilon_{w} \geq 0 \quad \text { for } 1 \leq w \leq N,
$$

where $N$ is the total number of the constraint points on the EXIT curve, indexed by $w$. Equation (17) can be further modified to:

$$
\begin{aligned}
& I_{e, \mathrm{Dec}, w}-I_{a, \mathrm{Dec}, w} \geq \varepsilon_{w} \geq 0 \\
& \Leftrightarrow \frac{\sum_{i=1}^{M} a_{i} \cdot d_{v_{i}} \cdot J\left(\sqrt{\left(d_{v_{i}}-1\right) \cdot J^{-1}\left(I_{e, D e m, w}\right)^{2}+J^{-1}\left(I_{e, C n d, w}\right)^{2}}\right)}{\sum_{i=1}^{M} a_{j} \cdot d_{v_{i}}}-I_{a, \text { Dem }, w} \geq \varepsilon_{w} \\
& \Leftrightarrow \sum_{i=1}^{M} a_{j} \cdot d_{v_{i}} \cdot\left(\sqrt{\left(d_{v_{i}}-1\right) \cdot J^{-1}\left(I_{e, D e m, w}\right)^{2}+J^{-1}\left(I_{e, C n d, w}\right)^{2}}\right)-I_{a, \text { Dem }, w} \sum_{i=1}^{M} a_{j} \cdot d_{v} \\
& \geq \varepsilon_{w} \cdot \sum_{i=1}^{M} a_{j} \cdot d_{v_{i}} \\
& \Leftrightarrow \sum_{i=1}^{M} a_{j} \cdot d_{v_{i}}\left(-J\left(\sqrt{\left(d_{v_{i}}-1\right) \cdot J^{-1}\left(I_{e, D e m, w}\right)^{2}+J^{-1}\left(I_{e, C n d, w}\right)^{2}}+I_{a, \text { Dem }, w}+\varepsilon_{w}\right)\right) \leq 0
\end{aligned}
$$

Therefore, (17) can be regarded as the linear function of $a_{i}$. Now the optimality criterion can be rewritten with the parameters and indexes defined above, as

$$
\begin{aligned}
& \text { Minimize } \sum_{i=1}^{M} a_{i} d_{v_{i}} \\
& \text { Subject to } \\
& \sum_{i=1}^{M} a_{i} \cdot d_{v_{i}}\left(-J\left(\sqrt{\left(d_{v_{i}}-1\right) \cdot J^{-1}\left(I_{e, D e m, w}\right)^{2}+J^{-1}\left(I_{\text {cnd,e, }, w}\right)}\right)+I_{a, D \text { Dem }, w}+\varepsilon_{w}\right) \\
& \quad \leq 0 \text { (for } 1 \leq w \leq N) \\
& \text { and } \sum_{i=1}^{M} a_{i}=1
\end{aligned}
$$

Now, given the fact that the optimization parameter in (19) is only $a_{i}$ and the other terms are constant and furthermore, the index and constraints are both linear function of the optimization variable $a_{i}$. Hence, the problem can be solved by using LP techniques.

\section{Appendix 2: Summary of BSA}

Labeling pattern shown in [13] for DSI-BICM-ID-EM is obtained by using binary switching algorithm (BSA) for optimization where labeling cost function defined in this section is used. In [6], labeling cost refers to pairwise error probability in AWGN channels with full a priori information. Assuming that the bit to be detected full $a$ priori MI for the rest of the $\ell_{\text {map }}-1$ bits is available, the average pairwise error probability $Z_{\ell_{\text {map }}-1}$ between the two symbols, originally transmitted symbol $s$ and the other symbols $\hat{s}$, is given by,

$$
Z_{\ell_{\text {map }}-1}=\frac{1}{\ell_{\text {map }} 2^{\ell_{\text {map }}}} \sum_{v=1}^{\ell_{\text {map }}} \sum_{s s_{v}=0} \sum_{\hat{s} \hat{s}_{v}=1} \exp \left(-\left|\mu\left(s_{v}\right)-\mu\left(\hat{s}_{v}\right)\right|^{2} / \sigma_{n}^{2}\right),
$$

where function $\mu(\cdot)$ returns the constellation point corresponding to the labeling patterns $s_{v}$ and $\hat{s}_{v}$ being the $v$ th bit being 0 and 1 . The average pairwise probability given by (20) is used as a cost function, which can be further decomposed as a sum of the cost functions for each fixed symbol $s^{h}$, given the fact that $Z_{\ell_{\text {map }}-1}=\sum_{h=0}^{2^{\ell_{\text {map }}-1}} Z_{\ell_{\text {map }}-1}^{h}$, where,

$$
Z_{\ell_{\operatorname{map}}-1}^{h}=\frac{1}{\ell_{\text {map }} 2^{\ell_{\text {map }}}} \sum_{v=1}^{\ell_{\text {map }}} \sum_{s^{h} \mid s_{v}^{h}=0} \sum_{s^{n} \mid s_{v}^{h}=1} \exp \left(-\left|\mu\left(s_{v}^{h}\right)-\mu\left(s_{v}^{\widehat{h}}\right)\right|^{2} / \sigma_{n}^{2}\right),
$$

for $h=0,1, \ldots, 2^{\ell_{\text {map }}}-1$. The BSA is summarized in Algorithm 3.

\section{Algorithm 3 Binary switching algorithm (BSA)}

\section{repeat}

Initialization: generate labeling pattern randomly.

Select the symbol $s_{\text {high }}$ which has the highest cost $Z_{\ell_{\text {map }}-1}^{h}$.

Find the symbol $s_{\text {low }}$ which can achieve maximum reduction of the total

$\operatorname{cost} Z_{\ell_{\text {map }}-1}$ by swapping the positions of $s_{\text {high }}$ and $s_{\text {low }}$.

if $s_{\text {low }}$ exists. then

Swap $s_{\text {high }}$ and $s_{\text {low }}$.

Update $Z_{\ell_{\text {map }}-1}$ according to the new labeling pattern.

else

Set the symbol with the second highest cost as $S_{\text {high }}$.

\section{end if}

until There is no pair of symbols to switch

A problem with this approach is, however, that the cost is calculated assuming the availability of a full $a$ priori information and thus, $Z_{\ell_{\text {map }}-1}$ affect only the right most point of the DemD $D_{\text {dacc }}$ EXIT curve, and it does not consider the matching between the demapper and decoder EXIT curves. This approach is reasonable only when the objective is to force the right-most point of the demapper curve to reach as close to the $(1.0,1.0) \mathrm{MI}$ point as possible. However, this article has already proposed the use of D-ACC which already makes it possible 
for the demapper EXIT curve to reach a point close enough to the $(1.0,1.0)$ MI point. In the subsection 4-D, a novel algorithm, EBSA, is introduced to obtain a labeling pattern aiming at better matching between the two curves, assuming the use of D-ACC.

\section{Appendix 3: Cost functions for ABSA and EBSA}

The cost definition commonly used by ABSA and EBSA are summarized below. Given $\ell_{\text {map }}$, the number of the known bits $q$ satisfies

$$
q=0,1, \ldots, \ell_{\text {map }}-1 \text {. }
$$

For the labeling cost $Z_{q}$ with the $q$ known bits having their a priori $\mathrm{MI}=1, Z_{q}$ can be expressed as

$$
Z_{q}=\frac{1}{\ell_{\text {map }} 2^{\ell_{\text {map }}-1} 2^{\ell_{\text {map }}-q-1}} \sum_{v=1}^{\ell_{\text {map }}} \sum_{s s_{v}=0 s \mid \hat{s}_{v}=1} \sum_{1} \exp \left(-\left|\mu\left(s_{v}\right)-\mu\left(\hat{s}_{v}\right)\right|^{2} / \sigma_{n}^{2}\right) .
$$

Furthermore, since $Z_{q}$ can be decomposed into symbol-wise cost $Z_{q}^{h}$, as

$$
Z_{q}=\sum_{h=0}^{2^{\ell \text { map }}-1} Z_{q}^{h}
$$

with

$$
Z_{q}^{h}=\frac{1}{\ell_{\operatorname{map}} 2^{\ell_{\operatorname{map}}-1} 2^{\ell_{\operatorname{map}}-q-1}} \sum_{v=1}^{\ell_{\operatorname{map}}} \sum_{s^{h} s_{v}^{h}=0} \sum_{s^{h} l_{v}^{h} h_{v}=1} \exp \left(-\left|\mu\left(s_{v}^{h}\right)-\mu\left(\hat{s}_{v}^{h}\right)\right|^{2} / \sigma_{n}^{2}\right) .
$$

The labeling cost matrix for $\ell_{\text {map }}$ is defined as an $\ell_{\text {map }}$ $\times 2$ map matrix, given by

$$
Z=\left[\begin{array}{ccccc}
Z_{0}^{0} & Z_{1}^{0} & \cdots & Z_{\ell_{\text {map }}-2}^{0} & Z_{\ell_{\text {map }}-1}^{0} \\
Z_{0}^{1} & Z_{1}^{1} & \cdots & Z_{\ell_{\text {map }}-2}^{1} & Z_{\ell_{\text {map }}-1}^{1} \\
& \multicolumn{4}{c}{} \\
Z_{0}^{2_{\text {map }}-1} & Z_{1}^{\ell_{\text {map }}-1} & \cdots & Z_{\ell_{\text {map }}-2}^{2_{\text {map }}-1} & Z_{\ell_{\text {map }}-1}^{2_{\text {map }}-1}
\end{array}\right]
$$

Note that the $\left(2^{\ell_{\text {map }}}-1\right)$ low of the matrix $\mathrm{Z}$ corresponds to the case where the all bits in the label are one, since the symbol-wise cost is measured from a bit in the pattern being zero, and the larger the $\left|\mu\left(s_{v}^{h}\right)-\mu\left(\hat{s}_{v}^{h}\right)\right|^{2}$ value, the lower the cost. Furthermore, there are no terms summed up in the second summation in (20) and (21). Hence, all the costs in the $\left(2^{\ell_{\text {map }}}-1\right)$ th low are zero, i.e,

$$
\begin{aligned}
Z^{\ell_{\text {map }}-1} & =\left[\begin{array}{llll}
Z_{0}^{2_{\text {map }}-1} & Z_{1}^{\ell_{\text {map }}-1} \cdots & Z_{\ell_{\text {map }}-2}^{\ell_{\text {map }}-2} Z_{\ell_{\text {map }}-1}^{2_{\text {map }}-1}
\end{array}\right]_{(27)} \\
& =\left[\begin{array}{llll}
0 & 0 & \cdots & 00
\end{array}\right]
\end{aligned}
$$

Now given $q, q=0,1, \ldots, 2^{\ell_{\text {map }}}-1$, the total cost vector $\hat{\mathbb{Z}}$ for each $q$ is calculated by simply summing the column vector of $Z$ as

$$
\begin{aligned}
& \hat{\mathbb{Z}}=\left[Z_{0} Z_{1} \cdots Z_{\ell_{\text {map }}-2} Z_{\ell_{\text {map }}-1}\right] \\
& =[1 \cdots 1]\left[\begin{array}{ccccc}
Z_{0}^{0} & Z_{1}^{0} & \cdots & Z_{\ell_{\text {map }}-2}^{0} & Z_{\ell_{\text {map }}-1}^{0} \\
Z_{0}^{1} & Z_{1}^{1} & \cdots & Z_{\ell_{\text {map }}-2}^{1} & Z_{\ell_{\text {map }}-1}^{1} \\
& & \vdots & & \\
Z_{0}^{\ell_{\text {map }}-1} & Z_{1}^{\ell_{\text {map }}-1} & \cdots & Z_{\ell_{\text {map }}-2}^{\ell_{\text {mam }}-1} & Z_{\ell_{\text {map }}-1}^{\ell_{\text {map }}-1}
\end{array}\right] \text { 28) } \\
& =\left[\sum_{h=0}^{2^{\ell_{\text {map }}-1}} Z_{0}^{h} \sum_{h=0}^{2^{\ell_{\text {map }}-1}} Z_{1}^{h} \cdots \sum_{h=0}^{2^{\ell_{\text {map }}-1}} Z_{\ell_{\text {map }}-1}^{h}\right]
\end{aligned}
$$

Those costs affect the shape of the demapper EXIT curve. Figure 6 shows an intuitive example for $\ell_{\text {map }}=5$.

Now weighting vector $\lambda$ is introduced to take into account the fact that which cost is important from the viewpoint of the matching between the two EXIT curves. The weighted cost $\bar{Z}$ (scaler) is defined by using the weight vector $\lambda$, as

$$
\bar{Z}=\hat{\mathbb{Z}} \lambda=\hat{\mathbb{Z}}\left[\lambda_{0} \lambda_{1} \cdots \lambda_{\ell_{\text {map }}-2} \lambda_{\ell_{\text {map }}-1}\right]^{t} .
$$

Also, using $\lambda$, symbol wise weighted cost vector $\overline{\mathbb{Z}}$ can be calculated as

$$
\overline{\mathbb{Z}}=Z \lambda=\left[\bar{Z}^{0} \bar{Z}^{1} \cdots \bar{Z}^{2^{\ell \text { map }}-1}\right]^{t},
$$

yielding

$$
\bar{Z}=\sum_{i=0}^{2^{l \operatorname{map}}-1} \bar{Z}^{i}
$$

\section{Endnotes}

${ }^{\text {a }}$ The proposed technique is also applicable to other BICM-ID techniques, so far as they use LP for degree allocation optimization. LDPC aided BICM-ID as well as irregular convolutional code aided BICM-ID belong to this class. This is the reason on why we call the technique proposed in this article "framework". ${ }^{\mathrm{b}} \mathrm{Com}$ bined use of D-ACC with BICM-ID itself was first proposed by [9]. The technique is also used in [12] to determine the optimal doping rate when it is combined with BSA. ${ }^{c} d_{c}$ has indirect effect to the LP optimization, but to the code rate. ${ }^{\mathrm{d}}$ Schreckenbach et al. [6] show that $N_{\max }=100$ is enough. ${ }^{\mathrm{e}}$ The range is defined as: $\operatorname{MI}\left(Z_{q}+\Delta Z_{\mathrm{q}}\right)$ for $q=0, \operatorname{MI}\left(Z_{q} \pm \Delta Z_{q}\right)$ for $1 \leq q \leq$ $\ell_{\text {map }}-2$ and $\operatorname{MI}\left(Z_{q}-\Delta Z_{q}\right)$ for $q=\ell_{\text {map }}-1$, where $\Delta=1 /\left(\ell_{\text {map }}-1\right)$.

\section{Acknowledgements}

This research was in part supported by the Japanese government funding program, Grant-in-Aid for Scientific Research (B) No. 20360168 and (C) No. 22560367, and in part by Finland distinguished professor program funded by Finnish National Technology Agency Tekes. The authors are highly 
thankful for valuable technical comments and suggestions given by Mr. Takehiko Kobayashi of Hitachi Kokusai Electric Inc. We also acknowledge Mr. Xin He of Information Theory and Signal Processing Lab., School of Information Science, JAIST for his valuable opinions and suggestions to improve the quality of this article.

\section{Author details}

'School of Information Science, Japan Advanced Institute of Science and Technology (JAIST), 1-1 Asahidai, Nomi, Ishikawa, 923-1292, Japan ${ }^{2}$ Center for Wireless Communication (CWC), University of Oulu, Oulu Fl-90014, Finland

\section{Competing interests}

The authors declare that they have no competing interests.

Received: 28 April 2011 Accepted: 9 February 2012

Published: 9 February 2012

\section{References}

1. C Berrou, A Glavieux, Near optimum error correcting coding and decoding: turbo codes. IEEE Trans Commun. 44, 1261-1271 (1996). doi:10.1109/ 26.539767

2. X Li, J Ritcey, Bit-interleaved coded modulation with iterative decoding. IEEE Commun Lett. 10, 169-171 (1996)

3. S ten Brink, Convergence behavior of iteratively decoded parallel concatenated codes. IEEE Trans Commun. 49, 1727-1737 (2001). doi:10.1109/26.957394

4. J Hagenauer, The EXIT chart-introduction to extrinsic information transfer in iterative processing, in Proceedings of the 12th Europ. Signal Proc Conference (EUSIPCO), Vienna, Austria, 1541-1548 (Sept. 2004)

5. S ten Brink, G Kramer, A Ashikhmin, Design of low-density parity-check codes for modulation and detection. IEEE Trans Commun. 52, 670-678 (2004). doi:10.1109/TCOMM.2004.826370

6. F Schreckenbach, N Gortz, J Hagenauer, G Bauch, Optimized symbo mappings for bit-interleaved coded modulation with iterative decoding, Global Telecommunication Conference (GLOBECOM'03), 6, San Francisco, USA, 3316-3320 (Dec 2003)

7. P Henkel, Extended mappings for bit-interleaved coded modulation, in Personal, Indoor and Mobile Radios Communications, Helsinki, Finland, 1-4 (Sept. 2007)

8. P Henkel, Doping of extended mappings for signal shaping, in Vehicular Technology Conference (VTC) Spring, Dublin, 1851-1855 (April 2007)

9. S Pfletschinger, F Sanzi, Error floor removal for bit-interleaved coded modulation with iterative detection. IEEE Trans Commun. 5, 3174-3181 (2006)

10. S ten Brink, Rate one-half code for approaching the shannon limit by 0.1 dB. Electron Lett. 36, 1293-1294 (2000). doi:10.1049/el:20000953

11. K Zeger, A Gersho, Pseudo-gray coding. IEEE Trans Commun. 38, 2147-2158 (1990). doi:10.1109/26.64657

12. Z Yang, Q Xie, K Peng, J Song, Labeling optimization for BICM-ID systems. IEEE Commun Lett. 14, 1047-1049 (2010)

13. D Zhao, A Dauch, T Matsumoto, BICM-ID using extended mapping and repetition code with irregular node degree allocation, in Vehicular Technology Conference (VTC) Spring, Barcelona, Spain, 1-5 (April 2009)

14. K Fukawa, D Zhao, A Tölli, T Matsumoto, Irregular repetition and single parity check coded BICM-ID using extended mapping -optimal node degree allocation-, in 2010 5th International ICST Conference on Communications and Networking in China (CHINACOM), Beijing, China, 1-6 (August 2010)

15. D Zhao, A Dauch, T Matsumoto, Modulation doping for repetition coded BICM-ID with irregular degree allocation, in ITG Wireless Smart Antenna (WSA), Berlin, Germany, pp. 1-5 (February 2009)

16. RMR Tee, L Hanzo, EXIT-chart aided near-capacity irregular bit-interleaved coded modulation design. IEEE Trans Commun. 8, 32-37 (2009)

17. L Szczecinski, H Chafnaji, C Hermosilla, Modulation doping for iterative demapping of bit-interleaved coded modulation. IEEE Commun Lett. 9(12), 1031-1033 (2005). doi:10.1109/LCOMM.2005.1576578

18. M Tuchler, R Koetter, A Singer: Turbo equalization, principles and new results. IEEE Trans Commun. 50, 754-767 (2010)

19. L Hanzo, Turbo Coding, Turbo Equalization and Space-Time Coding for Transmission over Fading Channels, (John Wiley and Sons Inc., New York, 2002)
20. K Anwar, T Matsumoto, Very simple BICM-ID using repetition code and extended mapping with doped accumulator, Wirel Personal Commun, (Springer, 2011)

21. F Schreckenbach, G Bauch, Irregular signal constellations, mappings and precoder, in International Symposium on Information Theory and its Applications (ISITA), Palma, Italy, 1-5 (2004)

22. S Lin, D Costello, Error Control Coding, 2nd edn. (Prentice-Hall Inc., Upper Saddle River, NJ, USA, 2004)

23. S Che, S Tong, Low-complexity LDPC coded BICM-ID with orthogonal modulations. Electron Lett. 45, 845-846 (2009). doi:10.1049/el.2009.0521

doi:10.1186/1687-1499-2012-40

Cite this article as: Fukawa et al:: EXIT-constrained BICM-ID design using extended mapping. EURASIP Journal on Wireless Communications and Networking 2012 2012:40

\section{Submit your manuscript to a SpringerOpen ${ }^{\circ}$ journal and benefit from:}

- Convenient online submission

- Rigorous peer review

- Immediate publication on acceptance

- Open access: articles freely available online

- High visibility within the field

- Retaining the copyright to your article

Submit your next manuscript at $\gg$ springeropen.com 